\title{
UMA APLICAÇÃO DA REGRESSÃO LOGÍSTICA NO INVENTÁRIO DE ESTILOS DE APRENDIZAGEM DE CANFIELD (LSI) SOB A ÓTICA DAS REPROVAÇÕES ACADÊMICAS
}

\author{
JACQUELINE VENEROSO ALVES CUNHA \\ Doutora em Ciências Contábeis pela \\ Universidade de São Paulo \\ Professora da Universidade Regional de Blumenau \\ E-mail: jvac@furb.br
}

\author{
EDGARD B. CORNACHIONE JUNIOR \\ Professor Livre-Docente da FEA/USP \\ Doutor em Ciências Contábeis pela FEA/USP \\ E-mail: edgardbc@usp. br
}

Resumo

\author{
GILBERTO DE ANDRADE MARTINS \\ Professor Livre-Docente da FEA/USP e \\ Professor Titular do Departamento de \\ Contabilidade e Atuária da FEA/USP \\ E-mail: martins@usp.br
}

A proposta deste estudo é testar as variáveis consideradas pelo Inventário de Estilos de Aprendizagem de Canfield (LSI) como preditoras da probabilidade de estabelecer o nível de repetência nos discentes dos Cursos de Ciências Contábeis no contexto brasileiro. Partindo do Inventário de Estilos de Aprendizagem de Canfield (LSI), que visa estabelecer o estilo de aprendizagem de estudantes, procura-se relacionar as dimensões do estilo de aprendizagem e a probabilidade das falhas acadêmicas. 0 questionário foi aplicado no $2^{\circ}$ semestre letivo de 2004, em uma das maiores instituições de ensino particulares do Estado de Minas Gerais, exclusivamente para os alunos do Curso noturno de Ciências Contábeis. Para atender ao objetivo estabelecido, utilizou-se a ferramenta estatística da regressão logística estimando o modelo de equação que melhor atendesse a esse propósito. A pesquisa compreendeu o exame dos dados de 177 estudantes. Com base nos dados pesquisados, a conclusão que se chega é que a reprovação está associada positivamente à preferência dos alunos por detalhes, por ouvir, quando se trata de aulas expositivas e por altas expectativas relativas às suas notas.

Palavras-chave: estilos de aprendizagem; reprovações; Ciências Contábeis.

\begin{abstract}
The proposal of this study is to test the variables considered by Canfield's learning styles inventory (LSI) as the probability predictive to establish the level of repetition of students in Accounting Courses in Brazilian context. Beginning with Canfield's learning styles inventory (LSI), which aims to establish the students' learning style, we seek to correlate the dimensions of the learning style and the probability of academic failures. The questionnaire was applied in the second semester of the school-year 2004 in one of the biggest institutions for private education in the state of Minas Gerais, exclusively for the students of the Accounting evening course. To fulfill the established objective, logistic regression statistic tool was used, estimating the equation model that would better fulfill this purpose. The research took into consideration the analysis of 177 students' data. Based on the data researched, the conclusion is that the disapproval is associated positively to the students' preference for the details, for hearing when expositive classes are considered and for their high expectations about their grades.
\end{abstract}

Key words: learning styles, disapproval, Accounting. 


\section{INTRODUÇÃO}

\subsection{PROBLEMA DE PESQUISA}

Nos últimos anos, o ensino de uma forma geral, e principalmente o ensino superior em Ciências Contábeis, passa por uma crise que pode ter em sua origem questões como: a quase totalidade de cursos noturnos, pouca preparação didática dos professores, má qualidade das instituições de ensino e abertura desordenada de instituições de ensino e de cursos.

De acordo com o banco de dados do MEC/INEP, em 2002, no Brasil, eram 641 cursos superiores de contabilidade (80,5\% deles oferecidos por instituições privadas). Em 2003, o número de cursos alcançou 701 (80,6\% nas privadas), representando um incremento de $9,4 \%$ em apenas um ano. 0 número de estudantes envolvidos nesses cursos expandiu de 147.475 em 2002 para 157.991 em 2003. Isso representa 4\% do total de estudantes de graduação do país. Tomando por base todo o ensino superior, em 2003 eram 268.816 pessoas exercendo funções docentes no país, sendo 110.020 delas com regime de trabalho horista e 96.326 em tempo integral (71.001 nas instituições públicas), e apenas 56.238 com curso de doutoramento (36.265 nas públicas).

É alarmante o alto número de repetências, influenciando negativamente a motivação de docentes e discentes, bem como, elevando os custos de formação dos estudantes. Esse quadro se agrava quando transposto para o grau de absenteísmo e abandono dos cursos. Conforme a pró-reitora de graduação da USP, professora Sônia Penin, os problemas do ensino básico se refletem no ensino superior (JORNAL DA USP, 2005). As taxas de reprovação se mostraram crescentes, tanto no nível médio (8\% e $9 \%$ ) quanto no fundamental (11\% e 11,7\%), nos anos de 2001 e 2002, respectivamente (MEC/INEP).

Buscar um entendimento sobre os mecanismos das reprovações é papel dos pesquisadores das áreas de ensino neste país e representa condição relevante para que se possa atuar com efetividade visando a melhoria do processo ensino-aprendizagem.

Ninguém ganha e ninguém deseja uma reprovação. Considerando que o discente perderá tempo e dinheiro, o professor ficará mais desgastado e a instituição de ensino terá problemas para administrar alunos em transições curriculares. (MAHON, 2005).

Esses estudos não têm intuito de banir a reprovação das escolas, mas o propósito de entender seu mecanismo e as responsabilidades envolvidas em sua ocorrência.

Um dos aspectos que deve ser observado é o que trata das diferentes formas de aprendizagem existentes. 0 estilo de aprendizagem pode ser fundamental quando se parte do pressuposto de que a avaliação e, conseqüentemente a aprovação, ou reprovação, são reflexos diretos do que é ensinado e, principalmente, do que é aprendido nas escolas. Assim, informações sobre os estilos de aprendizagem podem ajudar professores a tornarem-se mais sensíveis às diferenças que os estudantes apresentam em sala (MCKEACHIE, 2002), e serem úteis para que os alunos busquem formas de atender suas características de aprendizagem, privilegiando-as, ou até mesmo estimulando suas aptidões não tão latentes.

A proposta deste estudo é testar as variáveis estabelecidas pelo Inventário de Estilos de Aprendizagem de Canfield (LSI) como preditoras da probabilidade de estabelecer o nível de repetência nos discentes dos Cursos de Ciências Contábeis no contexto brasileiro. Partindo do Inventário de Estilos de Aprendizagem de Canfield (LSI), que visa estabelecer o estilo de aprendizagem de estudantes, procura-se relacionar as dimensões do estilo de aprendizagem e a probabilidade de falhas acadêmicas. 
Analisando a importância relativa às falhas acadêmicas apresenta-se a seguinte questão: existem preferências por um método específico de aprendizagem, dentre aquelas estabelecidas pelo Inventário de Estilos de Aprendizagem de Canfield (LSI), que influenciam a probabilidade de um aluno reprovar em uma disciplina?

Este estudo é desenvolvido com a finalidade de encontrar evidências que possam responder à questão abordada, contribuindo para o aumento do conhecimento sobre os estilos de aprendizagem e falhas acadêmicas.

Estabelece-se como hipótese de pesquisa que algumas preferências de aprendizagem dos discentes interferem na sua probabilidade de aprovação.

\subsection{OBJETIVO}

O objetivo deste artigo é verificar a existência de variáveis que influenciam a probabilidade associada à ocorrência de reprovação em disciplinas conforme as diversas percepções dos alunos do Curso noturno de graduação em Ciências Contábeis, de uma faculdade particular localizada em Belo Horizonte - MG, utilizando como variáveis independentes as questões estabelecidas no Inventário de Estilos de Aprendizagem de Canfield (LSI).

\subsection{JUSTIFICATIVA}

Tendo como parâmetro o número de estudos publicados no Brasil abrangendo a área educacional, principalmente quando se trata do Curso de Ciências Contábeis e, considerando-se a necessidade de identificar a probabilidade de um determinado aluno ser reprovado ao longo do seu processo de graduação para minimizar os efeitos já expostos, justifica-se plenamente a abordagem que se propõe.

Do ensino básico ao superior, os índices de evasão e repetência no Brasil são piores que nos países mais pobres da América Latina. 0 brasileiro adulto, em média, não tem seis anos de estudos - menos que panamenhos, equatorianos, colombianos e salvadorenhos. De cada 100 brasileiros entre 20 e 24 anos, apenas 12 estão numa faculdade. Esse número é inferior aos 20 observados no Chile, 30 no Uruguai e 40 na Argentina (RAINHO, 2005).

\section{REVISÃO DA LITERATURA}

\subsection{INVENTÁRIO DE ESTILOS DE APRENDIZAGEM DE CANFIELD (LSI)}

Dentre os modelos de estilos de aprendizagem preocupados com as preferências dos estudantes por um método específico de aprendizagem, o modelo de Canfield aparece presente em diversos estudos em campos distintos: sistema universitário público (DAVIS, 1979), estudantes mais velhos de ensino superior (WILLET e ADAMS, 1985), estudantes de enfermagem (MCCABE, 1983; MERRIT, 1983), terapia ocupacional (LLORENS \& ADAMS, 1978), educação física (PETTIGREW \& ZAKRAJSEK, 1984).

Esse modelo foi desenvolvido por um psicólogo com vasta experiência prática como consultor de instituições de ensino superior trabalhando para a melhoria das condições de 
aprendizagem estudantis (CLAXTON \& MURREL, 1987). A principal base teórica vem dos trabalhos de Maslow sobre a hierarquia das necessidades, e da pesquisa desenvolvida por McClelland sobre a motivação para o sucesso. Seu mérito é o uso de uma abordagem que não isola ensino e aprendizagem dentro de comportamentos separados (CANFIELD, 2000).

O Inventário de Canfield é considerado, no seu próprio manual, "um questionário de auto avaliação que permite aos estudantes descreverem que características de suas experiências educacionais eles preferem mais."i (CANFIELD, 2000, p. 1).

0 inventário é composto de 30 questões com 4 alternativas cada. Em cada uma dessas questões, o respondente estabelece suas preferências numa escala que varia de $1 \mathrm{a}$ 4, significando, respectivamente, do menor para o maior, a ordem do quão bem a alternativa corresponde à sua percepção no que se refere ao questionamento feito.

Canfield utiliza 20 variáveis explanatórias abrangendo as percepções dos alunos quanto aos aspectos abordados a seguir: formato das aulas de sua preferência, perfil dos professores do curso, relação mantida com os professores, afinidade com os colegas de curso, modo de estudo e expectativas quanto ao desempenho. Essas variáveis são divididas nos quatro grupos a seguir expostos.

\subsection{CONDIÇÕES DE APRENDIZAGEM}

Essa dimensão relata a preferência pela dinâmica da situação na qual ocorre a aprendizagem, incluindo afiliação (necessidades dos estudantes em desenvolver relações pessoais com colegas e professores), estrutura (necessidade por organização e detalhes), realização (vontade para estabelecer metas e ser independente), e superioridade (competição e orientação de autoridade). São oito as suas classificações:

- Peer (colegas): trabalhar em grupos de alunos; manter boas relações com outros alunos; ter alunos amigos, dentre outros. 0 aspecto mais importante da aprendizagem é o desenvolvimento e manutenção de entusiasmo, amizade, e relações interpessoais com indivíduos numa mesma situação ou grupo.

* Organization (organização): trabalho do curso organizado logicamente e com clareza, tarefas significativas e seqüência de atividades. O aspecto mais importante da aprendizagem é relacionado à seqüência de eventos e desenvolvimento de processos.

- Goal Setting (estabelecimento de objetivos): estabelecer os próprios objetivos, utilizar feedback para modificar os objetivos e procedimentos, tomar decisões próprias sobre objetivos.

- Competition (competição): desejo de comparação com os outros, necessidade de saber como está se saindo em relação aos outros. 0 indivíduo considera como relevante para uma aprendizagem efetiva as bases que permitem comparar entre seu desempenho com os dos outros.

- Instructor (professor): conhecer o professor pessoalmente, possuir entendimento mútuo, gostar um do outro. 0 indivíduo demonstra preferência por situações onde a amizade e as relações interpessoais com entusiasmo são mantidas entre estudante e professor.

- Detail (detalhes): informações específicas em tarefas, requisitos, regras. 0 indivíduo valoriza saber cada situação específica, com informação detalhada sobre o que é desenvolvido, como é feito, onde é feito e como será avaliado. 
* Independence (independência): trabalhar sozinho e de forma independente, determinar o plano de estudos próprio, fazer coisas para si mesmo. Preferência por conduzir tarefas por si mesmo e fazer coisas independentemente.

- Authority (autoridade): desejo de disciplina em sala de aula e manutenção da ordem, possuir professores informados e reconhecidos. Preocupação com o ambiente formal controlado e com autoridade. Preferem que os professores controlem o ambiente e os alunos.

\section{3 ÁREA DE INTERESSE}

Essa parte do instrumento lida com as preferências dos estudantes quanto ao assunto da matéria em termos de conteúdo ou objetos de estudo. Divide-se em quatro preferências.

* Numeric (numérico): trabalhar com números e lógica, computação, solução de problemas matemáticos. Preferência por estudos ou atividades de aprendizagem envolvendo computação, números e manipulação matemática, lógica e estatística.

- Qualitative (qualitativo): trabalhar com as palavras ou linguagem, escrever, editar, falar. Preferência por discussões diversas, críticas, onde diferentes pontos de vista e experiências possam ser desenvolvidos.

- Inanimate (inanimado): trabalhar com coisas. Construir, reparar, desenhar, operar. Preferência por envolvimento com itens relacionados a projetos, operar equipamentos e trabalhos manuais, simuladores.

- People (pessoas): trabalhar com pessoas, entrevistas, aconselhamento, vendas, ajuda ou assistência. Preferência por atividades envolvendo pessoas.

\subsection{MODO DE APRENDIZAGEM}

Essa dimensão se refere ao modo cognitivo que apóia a aquisição de novas informações. Abrange quatro preferências relativas à forma que a informação chega ao estudante.

- Listening (ouvir): ouvir informação; aulas expositivas, fitas, discursos. Preferência pelo canal auditivo em termos de aprendizagem.

- Reading (ler): examinar a palavra escrita; ler textos, panfletos. Preferência por aprender por meio de estudos de material impresso, livros, artigos.

- Iconic (icônico): ver ilustrações, filmes, slides, figures e gráficos. Preferência por atividades envolvendo qualquer meio gráfico ou representação de imagem.

- Direct Experience (experimentação direta): manusear ou desenvolver; loja, laboratório, trabalhos de campo, exercícios práticos. Preferência por aprender em situações com oportunidade de trabalhar com materiais, tópicos ou situações laboratoriais e de campo. 


\subsection{EXPECTATIVA DE NOTA}

Simplesmente reflete o nível de desempenho que o indivíduo espera de si mesmo. São cinco os níveis esperados.

- Expectativa A: o estudante assume fazer um trabalho superior ou excelente.

- Expectativa B: o estudante estima que seu desempenho seja acima da média ou bom.

- Expectativa C: a expectativa envolve um nível de desempenho médio.

- Expectativa D: representa uma expectativa de falha, com desempenho abaixo da média ou insatisfatório.

Sumarizando as dimensões e respectivas escalas consideradas no Inventário de estilos de aprendizagem de Canfield, apresenta-se a Tabela 1.

Tabela 1: Inventário de estilo de aprendizagem de Canfield

\begin{tabular}{c}
\hline Condições de aprendizagem (8 escalas) \\
Colegas \\
Organização \\
Estabelecimento de objetivos \\
Competição \\
Professor \\
Detalhes \\
Independência \\
Autoridade \\
Área de interesse (4 escalas) \\
Numérico \\
Qualitativo \\
Inanimado \\
Pessoas \\
Modo de aprendizagem (4 escalas) \\
Ouvir \\
Ler \\
Icônico \\
Experimentação direta \\
Expectativa de nota (4 escalas) \\
Expectativa A \\
Expectativa B \\
Expectativa C \\
Expectativa D
\end{tabular}

\section{METODOLOGIA}

Este trabalho configura-se numa abordagem empírico-analítica conforme a concepção de Martins (2000) que são as que apresentam em comum dentre outros, o uso de técnicas de coleta, tratamento e análise de dados marcadamente quantitativos e que privilegiam estudos práticos cuja prova de validação científica é buscada por meio de testes dos instrumentos. 
Com base na teoria apresentada foram analisadas variáveis para testar as preferências dos estudantes que possam afetar suas falhas acadêmicas, a saber: colegas (PEE), organização (ORG), estabelecimento de objetivos (GOA), competição (COM), professor (INS), detalhes (DET), independência (IND), autoridade (AUT), numérico (NUM), qualitativo (QUA), inanimado (INA), pessoas (PEO), ouvir (LIS), ler (REA), icônico (ICO), experiência direta (DIR), expectativa $A(E X A)$, expectativa $B(E X B)$, expectativa $C(E X C)$ e expectativa $D(E X D)$.

Em função da variável dependente (REP) - reprovação ser dicotômica, um grupo de alunos obteve reprovação em alguma disciplina, e outro não, a ferramenta estatística multivariada regressão logística foi empregada. Para se testar as preferências dos alunos que possam influenciar sua reprovação, será adotado o modelo representado pela equação a seguir:

$$
\begin{aligned}
& P(R E P)=\frac{1}{1+e^{-g(x)}} \quad \text { em que, } \\
& g(x)=\gamma_{0}+\gamma_{1} P E E+\gamma_{2} O R G+\gamma_{3} G O A+\gamma_{4} C O M+\ldots \ldots . .+y_{20} F A L
\end{aligned}
$$

A regressão logística é uma técnica estatística de análise multivariada utilizada em situações em que a variável dependente é dicotômica.

Essa técnica permite estimar a probabilidade que está associada à ocorrência de um determinado evento utilizando-se de um conjunto de variáveis independentes ou explicativas.

Segundo HAIR et al. (2005) a regressão logística encontra ampla aplicação nas situações nas quais o principal objetivo é identificar o grupo ao qual um item pertence (por exemplo, uma pessoa).

O uso da regressão logística é recomendado quando se apresentam restrições ao uso de outros modelos como, por exemplo, a análise discriminante. Essa técnica tem a capacidade de contornar pressupostos estabelecidos em outras técnicas, dentre eles, ausência de normalidade na distribuição dos erros, de linearidade entre as variáveis e de homogeneidade de variância de erros.

Diversos recursos computacionais encontram-se à disposição, atualmente, para estimar a equação de regressão logística. Neste estudo será utilizado o software SPSS $^{\circledR}$ em sua versão 10.0 .

\section{AMOSTRA E ANÁLISE DOS RESULTADOS}

Os dados foram coletados dentro de uma das maiores instituições particulares do estado de Minas Gerais, durante o segundo semestre acadêmico de 2004.

A instituição foi selecionada intencionalmente, ocasionando uma amostra por conveniência. A amostra foi composta de 235 estudantes de graduação, todos do Curso de Ciências Contábeis no período noturno, formalmente matriculados, e com pelo menos $70 \%$ de todos os créditos do programa já concluídos na data da coleta. formal.

Todas as participações foram voluntárias, e os alunos assinaram um consentimento 
Após excluir 58 respondentes não válidos, devido às falhas de preenchimento do questionário, um total de 177 questionários foi validado. 0 percentual de erros durante 0 preenchimento do questionário foi considerado alto, gerando $24,7 \%$ de respostas não válidas.

O questionário consiste de 2 grupos de questões: (i) um grupo composto de informações gerais sobre o participante ( 8 itens), e (ii) o instrumento de Inventário de Estilos de Aprendizagem de Canfield (30 itens).

0 instrumento Inventário de Estilos de Aprendizagem de Canfield foi comprado de seus desenvolvedores (Western Psychological Services), após contato e acordo de utilização por e-mail.

As variáveis mensuradas pelo primeiro grupo de questões são: $G_{0}$ (sexo), $G_{1}$ (paternidade), $\mathrm{G}_{2}$ (idade), $\mathrm{G}_{3}$ (ano de conclusão do ensino médio), $\mathrm{G}_{4}$ (outra graduação), $\mathrm{G}_{5}$ (experiência de trabalho - na área ou fora dela), $G_{6}$ (experiência em estágios - na área ou fora dela), $G_{7}$ (número de disciplinas repetidas).

A amostra era composta por $55 \%$ de homens. Em média, $69,4 \%$ dos respondentes não tinham filhos e a idade média era 29,6 anos.

Os estudantes haviam concluído o ensino médio durante o ano de 1993, em média, e apenas $5,1 \%$ da amostra responderam que já haviam concluído outro programa de graduação.

A grande maioria dos estudantes participantes $(95,6 \%)$ estava trabalhando, ou envolvidos em estágios. Sessenta e três vírgula três por cento dos respondentes nunca haviam repetido uma disciplina - 65 repetentes contra 112 não repetentes.

Considerando a segunda parte do questionário, os itens do Inventário de Estilos de Aprendizagem de Canfield, os participantes foram solicitados a ranquear a ordem de suas preferências em cada uma das quatro sentenças de cada item.

Analisando-se a correlação entre as variáveis (Tabela 2 ), verifica-se que a variável dependente REP - reprovação está correlacionada positivamente apenas com as variáveis independentes DET - detalhes, representando aquele indivíduo que valoriza saber cada situação específica, com informação detalhada sobre o que está desenvolvendo e a conseqüente avaliação que sofrerá; NUM - numérico, que caracteriza o discente que prefere trabalhar com números e lógica, computação e solução de problemas matemáticos; e EXA - expectativa A que reflete o nível de trabalho superior ou excelente que o indivíduo espera de si mesmo; e negativamente com EXC - expectativa $C$ quando a expectativa que o aluno tem de si mesmo é de um desempenho médio; e EXD - expectativa $D$ que reflete uma expectativa de fracasso pelo discente. 
Tabela 2 - Coeficientes de correlação de Spearman

\begin{tabular}{|c|c|c|}
\hline & & $R E P$ \\
\hline$R E P$ & Coeficiente de Correlação & 1,000 \\
\hline PEE & Coeficiente de Correlação & ,092 \\
\hline$O R G$ & Coeficiente de Correlação &, 057 \\
\hline GOA & Coeficiente de Correlação &,- 093 \\
\hline COM & Coeficiente de Correlação &,- 068 \\
\hline INS & Coeficiente de Correlação &,- 113 \\
\hline$D E T$ & Coeficiente de Correlação &, $186^{*}$ \\
\hline IND & Coeficiente de Correlação &,- 016 \\
\hline AUT & Coeficiente de Correlação &,- 002 \\
\hline NUM & Coeficiente de Correlação &, $182^{\star}$ \\
\hline QUA & Coeficiente de Correlação &,- 054 \\
\hline INA & Coeficiente de Correlação &,- 112 \\
\hline PEO & Coeficiente de Correlação &,- 044 \\
\hline LIS & Coeficiente de Correlação &,- 121 \\
\hline REA & Coeficiente de Correlação &,- 097 \\
\hline ICO & Coeficiente de Correlação & ,073 \\
\hline DIR & Coeficiente de Correlação &, 077 \\
\hline$E X A$ & Coeficiente de Correlação &, $290^{\star *}$ \\
\hline$E X B$ & Coeficiente de Correlação &, 076 \\
\hline EXC & Coeficiente de Correlação &,$- 246^{\star *}$ \\
\hline EXD & Coeficiente de Correlação &,$- 212^{* *}$ \\
\hline
\end{tabular}

As correlações com as outras variáveis independentes não se apresentaram significativas estatisticamente.

Com as respostas pertencentes a cada uma das quatro dimensões consideradas pelo instrumento foi estimada a equação envolvendo as avaliações das percepções dos alunos nas quatro dimensões originais em conjunto.

A validação estatística do modelo é feita por meio dos testes e indicadores apresentados na Tabela 3. 
Tabela 3 - Testes e indicadores de validação do modelo de regressão logística

\begin{tabular}{lc}
\hline \multicolumn{2}{l}{ Testes e Indicadores } \\
\hline Step, Block e Model & \\
\hline Estatística Qui-Quadrado & 35,431 \\
$p$-value & $0,002^{* * *}$ \\
-2LL & 197,312 \\
Nagelkerke R ${ }^{2}$ & $24,8 \%$ \\
Hosmer \& Lemeshow & \\
\hline Estatística Qui-Quadrado & 3,649 \\
p-value & $0,887^{* * *}$ \\
\hline
\end{tabular}

*** $p$-value é significativo a um nível de significância de $1 \%$

Os Testes Step, Block e Model são utilizados para avaliar a hipótese de nulidade de todos os coeficientes. Os resultados indicam rejeição da hipótese nula a um nível de significância de 10\%, 5\% e 1\% e comprovam a adequação do modelo. encontrado.

0 indicador -2LL informa o bom ajustamento geral do modelo pelo baixo valor

O poder explicativo do modelo é dado pelo indicador Nagelkerke $R^{2} ; 24,8,3 \%$ das variações registradas na variável dependente são explicadas pelo conjunto de variáveis independentes.

A precisão do modelo é avaliada pelo Teste Hosmer \& Lemeshow. Pode-se afirmar que inexistem diferenças significativas entre os resultados observados e os previstos pelo modelo.

Analisando-se os coeficientes da regressão evidenciados na Tabela 4, verifica-se que as variáveis DET - detalhes, LIS - ouvir e EXA - expectativa A são estatisticamente significativas ao nível de $10 \%$.

De modo geral, as variáveis cujos coeficientes são significativamente diferentes de zero explicam melhor as variações da variável dependente. 0 sinal positivo do coeficiente aumenta a probabilidade de ocorrência de reprovação. 
Tabela 4 - Coeficientes e resultados do Teste Wald

\begin{tabular}{|c|c|c|c|c|}
\hline \multirow{2}{*}{ Variáveis } & \multirow{2}{*}{$\begin{array}{c}\text { Sinal } \\
\text { enc ontrado }\end{array}$} & \multirow{2}{*}{ Coeficientes } & \multicolumn{2}{|c|}{ Teste Wald } \\
\hline & & & Estatística & p-value \\
\hline PEE & + & 0,078 & 1,020 & 0,312 \\
\hline ORG & + & 0,014 & 0,028 & 0,867 \\
\hline GOA & - & $-0,104$ & 1,783 & 0,182 \\
\hline INS & - & $-0,011$ & 0,027 & 0,870 \\
\hline DET & + & 0,161 & 3,255 & $0,071^{*}$ \\
\hline IND & + & 0,099 & 1,467 & 0,226 \\
\hline NUM & + & 0,077 & 1,477 & 0,224 \\
\hline QUA & - & $-0,033$ & 0,192 & 0,661 \\
\hline INA & - & $-0,014$ & 0,040 & 0,841 \\
\hline LIS & - & $-0,139$ & 4,793 & 0,029 ** \\
\hline REA & - & $-0,078$ & 2,015 & 0,156 \\
\hline ICO & - & $-0,004$ & 0,004 & 0,949 \\
\hline EXA & + & 0,235 & 5,639 & 0,018 ** \\
\hline EXB & + & 0,063 & 0,472 & 0,492 \\
\hline EXC & + & 0,041 & 0,123 & 0,726 \\
\hline Constant & - & $-5,722$ & 0,968 & 0,325 \\
\hline
\end{tabular}

Como as variáveis DET, LIS e EXA apresentaram resultados significativos estatisticamente não se pode rejeitar a hipótese estabelecida, isto é, algumas preferências de aprendizagem dos discentes interferem na sua aprovação. Dentre elas, o indivíduo que apresenta característica detalhista para informações específicas em tarefas, regras e requisitos, preferência por assistir a aulas expositivas, fitas e discursos e que assume ter a expectativa de um desempenho superior ou excelente.

\section{DELIMITAÇÃO DO ESTUDO}

Este trabalho delimitou-se ao estudo das preferências de aprendizagem dos estudantes baseado no Inventário de Estilos de Aprendizagem de Canfield (LSI).

Foram estudados os resultados do questionário aplicado no segundo semestre letivo de 2004, em uma das maiores instituições de ensino superior particulares do Estado de Minas Gerais, exclusivamente nos alunos do Curso noturno de Ciências Contábeis.

A amostra deste estudo foi obtida por meio de um método não-probabilístico, empregando a amostragem intencional devido ao acesso e propósitos do mesmo. Selltiz considera que

em uma amostra não probabilística, não há uma maneira de estimar a probabilidade que cada elemento tem de ser incluído na amostra, e não assegura que todos os elementos tenham alguma chance de ser incluído. ii (1959, p. 514)

\section{CONSIDERAÇÕES FINAIS E SUGESTÕES PARA PESQUISAS POSTERIORES}

Após a revisão da literatura e das análises que apoiaram este estudo, foram reunidas evidências que possibilitaram informação útil em termos de um melhor 
entendimento dos problemas relacionados à educação superior nos Cursos de Ciências Contábeis no Brasil, que foram a meta dessa pesquisa.

Antes de iniciar as discussões das constatações da pesquisa é crucial esclarecer que, devido à característica de não aleatoriedade da amostra, os resultados aqui relatados não poderão ser estendidos a outras amostras ou à população geral. Servirá, entretanto, como importante balizador e parâmetro para estudos posteriores nessa, e em outras áreas.

A resposta à questão de pesquisa aqui estabelecida, considerando graduandos em Ciências Contábeis de uma instituição de ensino privada de uma das maiores instituições privadas do Brasil, passa a ser apresentada a seguir.

Existem preferências por um método específico de aprendizagem, dentre aquelas estabelecidas pelo Inventário de Estilos de Aprendizagem de Canfield (LSI), que influenciam a probabilidade de um aluno reprovar em uma disciplina?

Considerando a questão de pesquisa, constatou-se que as variáveis Detalhes (DET), Ouvir (LIS) e Expectativa A (EXA) apresentaram coeficientes significativamente diferentes de zero no teste Wald, sugerindo que tais variáveis influenciam a probabilidade de um aluno reprovar em uma disciplina no Curso de Ciências Contábeis. Ou seja, de acordo com - LSI, os discentes com as características a seguir aumentam a probabilidade de reprovação: discentes cuja condição de aprendizagem é detalhista, que valorizam saber cada situação específica, com informação detalhada sobre o que é desenvolvido, como é feito, onde é feito e como será avaliado; cujo modo de aprendizagem preferido seja pelo canal auditivo com aulas expositivas, fitas, discursos; e, ainda aquele estudante que assume fazer um trabalho superior ou excelente relativo a sua expectativa de nota.

O conjunto de características que influenciam positivamente a probabilidade de reprovação sugere a ineficiência de uma metodologia de aula utilizada regularmente nas faculdades. Além de ressaltar a ineficácia da preocupação detalhista e mostrar que expectativas de um desempenho excelente são prejudiciais.

Após responder a questão de pesquisa proposta, o estudo sugere um baixo poder preditivo das dimensões utilizadas no Inventário de Estilos de Aprendizagem de Canfield (LSI) quando a variável dependente é a ocorrência ou não de repetência nos alunos do Curso noturno de Ciências Contábeis de uma instituição de ensino particular.

Estudos adicionais em outras instituições de ensino superior, públicas ou particulares, devem ser realizados e a comparação dos resultados, em estudos baseados no Inventário de Estilos de Aprendizagem (LSI), ou em outros, pode trazer ajuda adicional ao principal objetivo que deve ser perseguido pelos educadores, instituições e discentes deste país: a melhoria contínua das condições de ensino.

\section{REFERÊNCIAS}

CANFIELD, A. A. Learning styles inventory (LSI) Manual, $3^{\text {rd }}$ printing. Los Angeles: Western Psychological Services, 2000.

CLAXTON, C. S. \& MURRELL, P. H. Learning styles: implications for improving educational practices. ASHE-ERIC Higher Education Report n. 4. Washington: Association for the Study of Higher Education, 1987.

DAVIS, C. L. The interaction of learning and instructional styles as related to developmental studies student achievement. Unpublished doctoral dissertation, University of Georgia, 1979. 
HAIR JR., Joseph F., ANDERSON, R. E., TATHAM, Ronald L. Black, WILLIAN. Multivariate Data Analysis: with Readings. 5.ed. New Jersey: Prentice Hall, 1998.

Ensino básico não pode ser ignorado. Jornal da USP. Ano XX, nº 727, jun. 2005.

LLORENS, L. A. \& ADAMS, S. P. Learning style preferences of occupational therapy students. American Journal of Occupational Therapy, 1978, 32, v. 3, p. 161-164.

MAHON, E. A hora dos ruminantes: o que é uma reprovação? Disponível em << http://www.anup.com.br/txt/artigos/007_artigo.htm.>>. Acesso em 13 jun. 2006.

MARTINS, G. A. Manual para elaboração de monografias e dissertações. 2.ed. São Paulo: Atlas, 2000.

MCCABE, M. J. Analysis of learning styles of nursing students at University of Central Florida. Curriculum and Program Planning. Practicum paper, Nova University. ERIC Document Reproduction Service no. ED 249834, Office of Educational Research and Improvement, U.S. Department of Education, 1983.

MCKEACHIE, W. J. McKeachie's teaching tips: strategies, research, and theory for college and university teachers. $11^{\text {th }}$ ed. New York: Houghton Mifflin, 2002.

MEC (Ministério da Educação). Evolução do ensino superior - graduação 1980-1998. Brasília: INEP. Disponível em <<http://www.inep.gov.br/download/censo/1998/superior/evolucao_1980998.zip>> Acesso em 18 jun. 2005.

MEC (Ministério da Educação). Resultados e tendências da educação superior no Brasil. Brasília: INEP. Disponível em <<http://www.inep.gov.br/download/censo/2000/Superior/brasil.pdf >>. Acesso em 18 jun. 2005.

MEC (Ministério da Educação). Sinopse da educação superior 2002. Brasília: INEP. Disponível

em <<http://www.inep.gov.br/download/censo/2002/sinopse/Sinopse_2002_censosuperior.zi p >> Acesso em 18 jun. 2005.

MEC (Ministério da Educação). Sinopse da educação superior 2003. Brasília: INEP. Disponível em <<http://www.inep.gov.br/download/superior/2004/SES2003.zip>>. Acesso em 20 jun. 2005.

MEC (Ministério da Educação). Sistema de estatísticas educacionais. Brasília: INEP. Disponível em <<http://www.edudatabrasil.inep.gov.br/branco.html >>. Acesso em 20 jun. 2005.

MERRIT, S. L. Learning style preference of baccalaureate nursing students. Nursing Research, 1983, 32, 6, p. 367-372.

PETTIGREW, F. E. \& ZAKRAJSEK, D. A profile of learning style among physical education majors. Physical Educator, 1984, 41, 2, p. 85-89.

RAINHO, J. M. Pela porta dos fundos. Disponível em <<http://www2.uol.com.br/aprendiz/n_revistas/revista_educacao/setembro01/capa.htm> $\geq$. Acesso em 14 jun. 2005.

SELLTIZ, C.; JAHODA, M.; DEUTSCH, M.; Cook, S. W. Research methods in social relations. New York: Holt, Rinehart and Wanston, 1959.

WILLET, L. H.; ADAMS, F. G. Learning style preferences of external degree students. Innovative Higher Education, 1985, 10, 1, p. 55-65. 
